\title{
Patella Locking Star-Plate as Salvage to the Failed Tension Band Wiring for a Patella Fracture: A Case Report
}

\author{
Hemant Bansal Abhijit Behera Vijay Sharma Kamran Farooque \\ Department of Orthopaedics, JPNATC, AlIMS, New Delhi, India
}

Keywords

Failed P Patella fracture $\cdot$ Tension band $\cdot$ wiring $\cdot$ Patella plate

\begin{abstract}
The integrity of the patella is pivotal for the efficient functioning of the knee extensor mechanism. Thus, patella fracture management cannot be neglected and utmost priority should be determined for its preservation and maintenance of knee functioning. Tension band wiring (TBW) is the most widely and commonly used mode of fixation of patella fractures with consistently favourable outcomes. However, the complications associated with the same could be challenging and may compromise the integrity of the patella. This case report highlights the salvage of the failed TBW for a patella fracture with biomechanically stronger angular stable Star-shaped patella plate.
\end{abstract}

\section{Introduction}

Patella fracture is one of the common fractures in the lower limb [1]. It can be an isolated fracture or in association with adjacent long bones fracture. Patella forms an integral part of the knee extensor mechanism and is essential to keep the knee biomechanics aligned for independent ambulation [2]. Thus, active management of fracture in the patella need not be neglected and utmost priority should be determined towards the preservation of this important biomechanical pivotal structure.

In the majority, common displaced transverse, two-part, patella fractures are managed with tension band wiring (TBW) using Kirschner (K) wires or cannulated screws [3]. Though, 

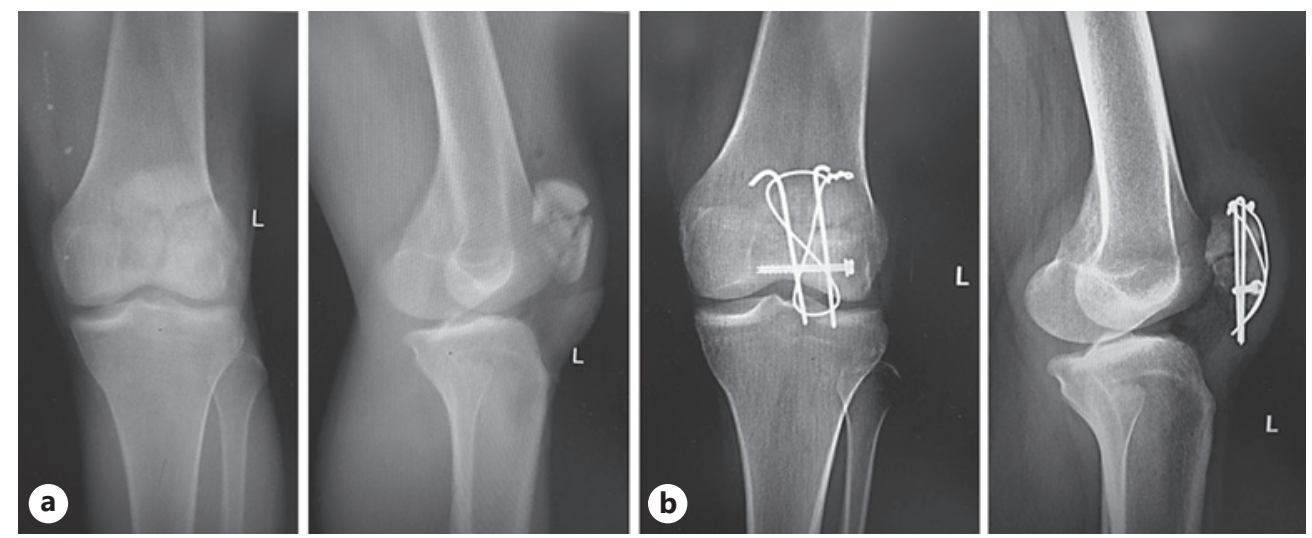

Fig. 1. a Preoperative radiographs showing three-part, T-shaped patella fracture left side. $\mathbf{b}$ Postoperative radiographs showing TBW and $4 \mathrm{~mm}$ CCS.

the principle of fixation remained the same, various innovations and modifications have been added to this conventional method of fixation of the patella fracture [4].

Patella fractures with comminution or more than two major fragments added technical challenges in their fixation, especially using the conventional tension band wiring method. However, if the principle of TBW when followed and applied properly can result in favourable outcomes [5]. Recently, patella locking plates have been designed to tackle this challenge which allows multiple locking screws along the perimetry of the patella, thus maintaining the reduction and allowing early rehabilitation compared to the conventional methods [6]. The present case report highlights the merits of the patella locking plate in a case of failed TBW in a patella fracture. The patient was informed that data concerning the case would be submitted for publication, and he provided consent.

\section{Case Description}

A 26-year-old man presented in the emergency department with pain, swelling in the left knee, and inability to bear weight on the affected limb with painful knee movements following a fall from a motorbike. On examination, he had contusion over the anterior aspect of the knee with diffuse swelling and tenderness. He was unable to actively extend the knee from the semi-flexed knee position. The limb was immediately immobilized, and other associated injuries were ruled out.

The radiographs of the affected knee joint revealed a displaced, fractured patella AO/ OTA $34 \mathrm{C} 2$ with a configuration of T-shaped fracture pattern, where the lower half of the patella was longitudinally split into two (shown in Fig. 1a). The affected knee joint was immobilized, and the limb was elevated along with local cold fomentation to allow early settling of the soft tissue around the fracture. After an interval of 3 days, once the wrinkling over the skin of the knee joint appeared, the patella fracture was operated using midline longitudinal incision, and firstly, the lower half of the fracture was reconstructed into a single fragment using a $4 \mathrm{~mm}$ Cannulated Cancellous Screw (CCS) and then the conventional TBW with K-wires and steel wires was done with anatomical articular reduction (shown in Fig. 1b).

Following surgery, the patient was given a knee immobilizer of 2 weeks and then gradual passive followed by active knee bending was started along with quadriceps strengthening exercises. Weight-bearing with support, as tolerated was also allowed. At the

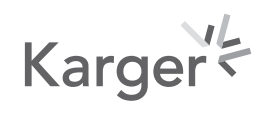


Case Reports in Orthopedic Research
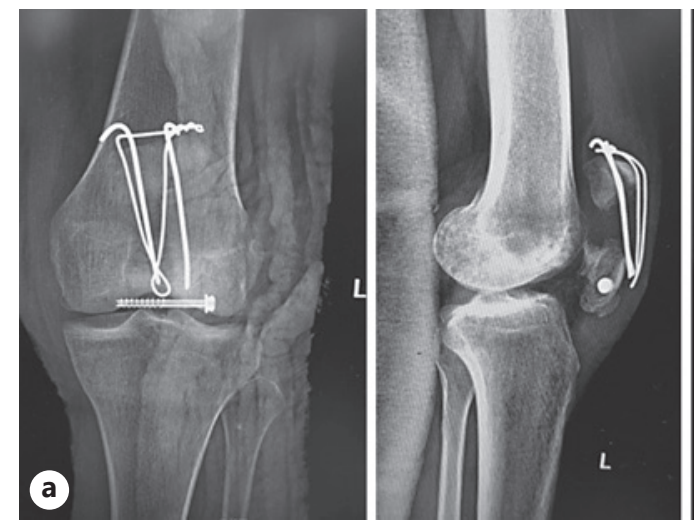
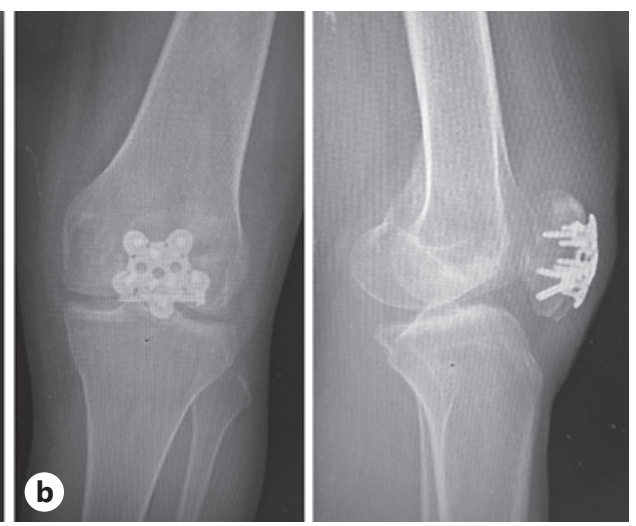

Fig. 2. a Radiographs showing cutout of TBW following re-trauma to the same, previously operated knee. The lower fragment was maintained as a single fragment with CCS in situ. b Postoperative radiographs showing fixation of the patella fracture with star-shaped locking patella plate.

follow-up of 6 weeks, he had full active range of motion in the treated knee joint and was allowed full weight-bearing without aid.

Two weeks later, the patient had an accidental slip from the stairs and sustained an injury to the same, previously operated knee joint. On radiographic assessment, he found to have cutout of the TBW construct from the lower half of the patella with grossly redisplaced patella fracture (shown in Fig. 2a). However, the lower half of the patella fixed using CCS maintained to be a single fragment.

The patient was again taken up for the surgery; the cut-out TBW was removed. Intraoperatively, the lower half was united and remained as a single fragment with CCS in situ. However, the anterior, central cortex was breached out with cut-out K-wires with a hollow cavity in the lower fragment. Thus, we opted to fix this state of the patella fracture with the medium-sized patella locking plate $\left(\right.$ Arthrex $\left.^{\circledR}\right)$, which allowed multiple locking screws to be inserted in the relatively dense peripheral part of the lower fragment. To augment the hollow cavity in the lower fragment and to enhance fracture union, autologous iliac crest grafting was also done (shown in Fig. 2b).

In the postoperative period, the knee was immobilized for 2 weeks and then gradual knee bending was started with quadriceps strengthening exercises, over the period of the next 4 weeks, and then full weight-bearing with support was started. The patient had fully recovered knee joint at the follow-up of 3 months with a united fracture. At 1-year follow-up, the patient had full knee range of motion with Oxford Knee Score of 46, without any complications related to the surgery or the hardware impingement (shown in Fig. 3).

\section{Discussion}

Fixation of the patella fractures with plates is an advancement in its surgical management. Wild et al. [7] first described the biomechanical aspect of the use of bilateral (along the medial and lateral border) poly-axial $2.7 \mathrm{~mm}$ plates in the surgical fixation of transverse patella fractures. Later, various biomechanical studies have proven these plates to be superior in stabilizing the fracture more securely as compared to the conventional tension band fixation methods $[6,8]$. Nevertheless, over the decade various plate designs and profiles have been used to internally fixed the patella fracture with favourable outcomes [9].

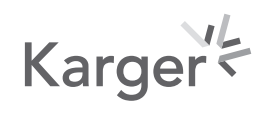



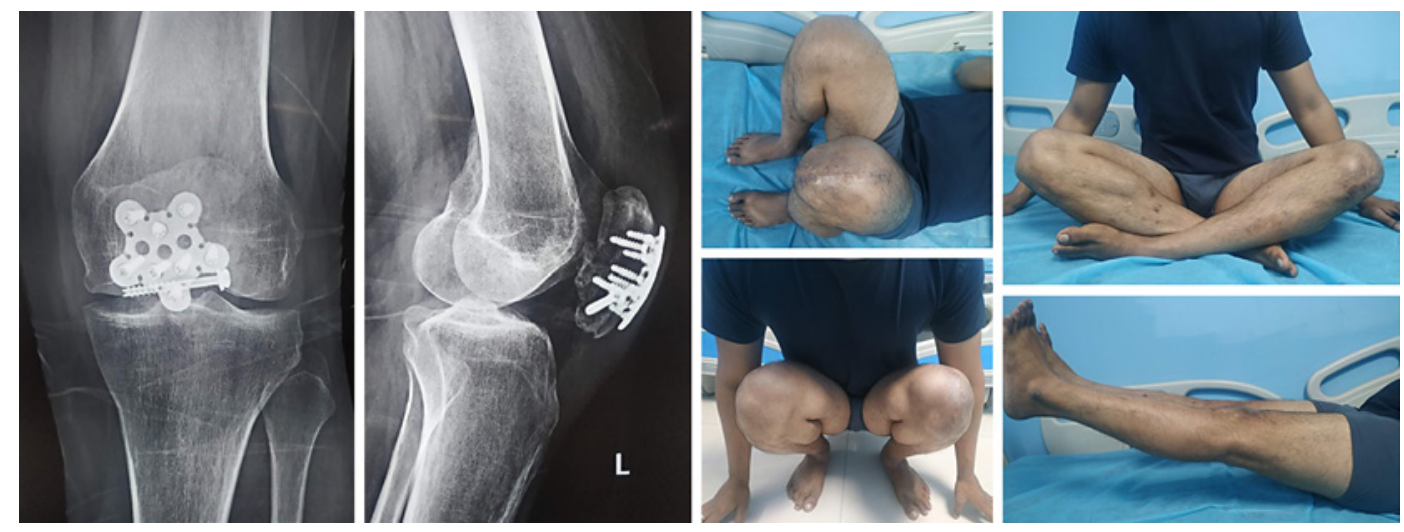

Fig. 3. One-year follow-up of the patient with well, united patella fracture with excellent functional outcomes without any implant-related complications.

The anterior locking patella plate $\left(\operatorname{Arthrex}^{\circledR}\right)$ is the popular one $[10,11]$, which is available in two configurations: the arrow-plate and the star-plate. Both plates are anatomically shaped and provide unidirectional angular-stability as well as sutures holes for the fixation of soft tissue to the plate. The arrow-plate is primarily indicated for simple tow-part fractures whereas; the star-plate is used for more comminuted fractures.

This angular stable patella plate provides better mechanical stability for the fracture fixation and is also devoid of complications related to the hardware impingement as the anatomical, low profile design fits well on to the anterior the surface of the patella and does not require a second surgery for implant removal once the fracture is united. Besides, the comminuted patella fractures or fractures with the osteoporotic bone are likely to benefit from the locking mechanism [10].

In the present case, the three-part, T-shaped patella fracture was initially fixed using the conventional method of TBW with good anatomical reduction and outcomes till the initial 6 weeks, following which the patient had accidental trauma to the same knee joint leading to cut out of the TBW with fracture displacement, which implies relatively inferior biomechanical strength of TBW as compared to the angular stable locking plate and the same has been proven in the several biomechanical studies $[6,7,9]$. This cutout had led to the deficient anterior cortex of the lower segment of the patella with central bone loss. Reconstructing the same with TBW would have been a compromised fixation, thus taking the advantages of the angle stable locking patella plate, the patella was fixed again anatomically with better biomechanical stability. As it was a revision surgery, the rehabilitation was delayed for the initial 2 weeks, though at the final follow-up patient had intact, united patella with full functioning. This case brings out the importance of keeping surgeons updated with the recent advancement in fracture management and also providing better alternatives for fracture fixation and patient care.

\section{Statement of Ethics}

Written informed consent was obtained from the patient for publication of this case report, and written permission was also obtained for possible printing and circulation of the accompanying radiological images solely for educational and academic purposes. This case report did not require ethical approval in accordance with Institute Ethics Committee, AIIMS, New Delhi guidelines. 


\section{Conflict of Interest Statement}

On behalf of all authors, the corresponding author states that there is no conflict of interest to disclose.

\section{Funding Sources}

No funding or grants were received or will be received from any commercial party relating to the subject of this article.

\section{Author Contributions}

The followings are the authors' contributions: conceptualization: [Hemant Bansal]; design: [Hemant Bansal, Abhijit Behera]; formal analysis and interpretation: [Hemant Bansal]; writing - original draft preparation: [Hemant Bansal]; writing - review and editing: [Hemant Bansal, Vijay Sharma]; and supervision: [Vijay Sharma, Kamran Farooque].

\section{Data Availability Statement}

All data generated or analysed during this study are included in this article. Further enquiries can be directed to the corresponding author.

\section{References}

Dietz SO, Hessmann MH, Gercek E, Rommens PM. Patellafraktur. Orthop Traumatol. 2009 Jun 1;21(2):206-20.

2 Kettelkamp DB. Clinical implications of knee biomechanics. Arch Surg. 1973 Sep 1;107(3):406-10.

3 Ruedi TP, Murphy WM. AO principles of fracture management. InAo Principles of Fracture Management; 2000. p. 868.

4 Lefaivre KA, O’Brien PJ, Broekhuyse HM, Guy P, Blachut PA. Modified tension band technique for patella fractures. Orthop Traumatol Surg Res. 2010 Sep 1;96(5):579-82.

5 Eggink KM, Jaarsma RL. Mid-term (2-8 years) follow-up of open reduction and internal fixation of patella fractures: does the surgical technique influence the outcome? Arch Orthop Trauma Surg. 2011 Mar 1;131(3): 399-404.

6 Thelen S, Schneppendahl J, Jopen E, Eichler C, Koebke J, Schönau E, et al. Biomechanical cadaver testing of a fixed-angle plate in comparison to tension wiring and screw fixation in transverse patella fractures. Injury. 2012 Aug 1;43(8):1290-5.

7 Wild M, Eichler C, Thelen S, Jungbluth P, Windolf J, Hakimi M. Fixed-angle plate osteosynthesis of the patella: an alternative to tension wiring? Clin Biomech. 2010 May 1;25(4):341-7.

8 Wild M, Fischer K, Hilsenbeck F, Hakimi M, Betsch M. Treating patella fractures with a fixed-angle patella plate: a prospective observational study. Injury. 2016 Aug 1;47(8):1737-43.

9 Dickens AJ, Salas C, Rise L, Murray-Krezan C, Taha MR, DeCoster TA, et al. Titanium mesh as a low-profile alternative for tension-band augmentation in patella fracture fixation: a biomechanical study. Injury. 2015 Jun 1; 46(6):1001-6.

10 Wurm S, Bühren V, Augat P. Treating patella fractures with a locking patella plate: first clinical results. Injury. 2018 Jun 1;49 Suppl 1:S51-5.

11 Ellwein A, Lill H, DeyHazra RO, Smith T, Katthagen JC. Outcomes after locked plating of displaced patella fractures: a prospective case series. Int Orthop. 2019 Dec;43(12):2807-15.

\section{Karger'}

